\title{
The experimental infection with a field isolate of the infectious bronchitis virus from eastern Saudi Arabia resulted in seroconversion of the challenged birds with no apparent clinical diseases
}

\author{
Maged Gomaa Hemida ${ }^{1,2}$ (D) Mohamed Al-Hammadi $^{1} \cdot$ Cecillio Gonzalves $^{1} \cdot$ \\ Mahmoud Moussa Ismail ${ }^{3}$
}

Received: 24 July 2020/Accepted: 18 January 2021/Published online: 8 April 2021

(C) Indian Virological Society 2021

\begin{abstract}
The infectious bronchitis virus (IBV) is still one of the major respiratory viral pathogens of chickens. The IBV infection resulted in a wide range of clinical syndromes in the affected chickens, including respiratory, renal, gonads affections as well as generalized infections. Despite the intensive application of various commercial vaccines against the virus, many outbreaks are still reported in chickens worldwide. Several studies reported the circulation of several strains and genotypes of the IBV in eastern Saudi Arabia. The main goal of the current study was to isolate some of the circulating strains of IBV and assess its ability to reproduce the IBV infections in the challenge birds. Another objective was to monitor the immune status of the infected chickens during the course of this study. To achieve these goals, we used some filed IBV isolates retrieved from an outbreak in a broiler chicken farm in eastern Saudi Arabia in 2014. A total of 220-dayold chickens (110 Ross and 110 native Saudi breed chickens), twenty birds per each group, were used in this study. The chickens in some groups received some IBV vaccines on day one of the experiment, and some are boosted on day 19. All birds were challenged on day 28 of the experiment. Our results showed mild IBV signs in the non-vaccinated control group of chickens; however, the
\end{abstract}

Maged Gomaa Hemida

mhemida@kfu.edu.sa

1 Department of Microbiology, College of Veterinary Medicine, King Faisal University, Alhuffuf, Al-Ahsa, Saudi Arabia

2 Department of Virology, Faculty of Veterinary Medicine, Kafrelsheikh University, Kafrelsheikh, Egypt

3 Department of Poultry Diseases, Faculty of Veterinary Medicine, Kafrelsheikh University, Kafrelsheikh, Egypt vaccinated chickens did not show any signs of IBV infections. Meanwhile, both the vaccinated and the nonevaccinated birds seroconverted to the IBV as shown by the ELISA results. In conclusion, the response of the IBV infected birds is mainly driven by the vaccination plans they received as a prime-boost regime. Further studies are required for a better understanding of the dynamics of IBV infection in native Saudi chickens.

Keywords IBV · Field strains · Challenge · Vaccine · ELISA - Seroconversion

\section{Introduction}

The IBV belongs to group III coronaviruses, which mainly infect various species of birds [1]. The virus is endemic in many regions throughout the world, including the Middle East, North and South Africa, as well as Latin America [2-5]. The IBV belongs to the family Coronaviridae and the order Nidovirales. The viral genome is single-stranded positive-sense RNA molecules [6]. The absence of the cross-protection among most IBV strains makes the preparation of vaccines against the IBV quite challenging [7]. The IBV infection results in both high morbidity and mortality rates up to $100 \%$ among the affected flocks [7]. The viral infection resulted in a wide range of clinical signs, including respiratory manifestations, nephritis, as well as pathological changes in the reproductive tracts of the affected birds [7]. The embryonated chicken eggs representing an ideal system for the isolation and propagation of many avian coronaviruses, including IBV, turkey coronavirus, and Pheasants coronavirus [8]. Likewise, other coronavirus candidates, the IBV genome is always prone to recurring charges on the genomic level due to the 
poor proofreading capabilities of their RNA dependent RNA polymerase [9]. This is in addition to the possibility of recombination among the various strain of the IBV [10]. These factors may contribute at least in part to the emergence of new strains, genotypes, and lineages of the IBV. In Saudi Arabia, several genotypes of the IBV were detected in many farms especially in the eastern region $[9,11,12]$. This is despite the massive application of various types of vaccines [9, 12]. Some virulent strains were detected in eastern Saudi Arabia which was responsible for high morbidity and mortality among the affected farms [12]. The IBV genotypes vary in their pathogenicity in chickens [11]. Some of the recently reported IBV lineages in chickens from eastern Saudi Arabia are related to vaccine strains such as the 4/91 and Mass lineages [11]. The main goal of the current study was to evaluate the efficacy of the filed isolated IBV strains from chickens in eastern Saudi Arabia to infect young chickens and to monitor the immune status of these birds during the course of the viral infections in these birds.

\section{Materials and methods}

\section{Animal ethics statement}

We conducted this study according to the guidelines of the Animal Ethics protocols and the National Committee of Bio-Ethics, King Abdul-Aziz City of Science and Technology, Royal Decree No. M/59 (http://www.kfsh.med.sa/ KFSH_WebSite/usersuploadedfiles\%5CNCBE\%20Regula tions\%20ENGLISH.pdf). Meanwhile, the sampling protocol was approved by the King Faisal University research ethics committee.

\section{Tissues and organs collection}

We collected specimens from some broiler chicken farms that suffered from acute respiratory manifestations in eastern Saudi Arabia. We selected some affected birds showing rales and nasal discharges. We conducted the necropsy examination of the selected chicken. We collected at least $5 \mathrm{~g}$ per each target organ (bronchi, trachea, lungs, kidneys, thymus, and cecal tonsils). Organs from each bird pooled into one tube and processed as a single sample. We prepared $10 \%$ tissue suspensions per each pooled sample as previously described [12]. The prepared tissue suspensions were subjected to two subsequent filtration steps through 0.45 then $0.22 \mu \mathrm{m}$ filters. The filtrated tissue suspensions were stored at $-80 \mathrm{C}$ for testing.

\section{Extraction of the viral RNAs}

The total viral RNAs were extracted from the pooled tissue samples from each bird as previously described [12]. Briefly, $140 \mu \mathrm{l}$ of each pooled sample was used to extract the total viral RNAs using the QIAamp Viral RNA Mini Kit kits (Qiagen, Germany). The extracted RNAs were eluted into $50 \mathrm{ul}$ elution buffer then stored at $-80{ }^{\circ} \mathrm{C}$ until further testing. The concentration of each RNA sample was measured by the RNA Nanodrop machine.

\section{Oligonucleotides}

We used the partial PPRV-N gene primers to confirm the detection of the IBV-RNA in the tested organs and tissues. The sequence of these primers as follows (IBV-NF-5'AATTTTGGTGATGACAAGATGA- $3^{\prime}$ and the IBV-NR5' CATTGTTCCTCTCCTCATCTG-3').

\section{The cDNA synthesis and the RT-PCR technique}

We used the RT-PCR technique to confirm the identity of the IBV isolates from chickens in eastern Saudi Arabia in 2015 .

\section{Isolation of the IBV strains}

We used the native breed embryonated chicken eggs for the isolation of the local isolates of IBV in eastern Saudi Arabia. This isolation process was carried out as previously described [12]. We conducted three blind passages of the IBV using the native breed of non-vaccinated chickens. We used the seeds of the passage- 3 for the virus challenge study. The virus seeds were subjected to the subsequent double filtration as described above. We tested the source of the native breeds and eggs to ensure there are no antibodies against the IBV in sera or egg yolk by the commercial IBV ELISA as described below.

\section{Commercial vaccines}

We used two commercial IBV vaccines in the chicken experiments (Nobilis ${ }^{\circledR}$ 4/91 (IB-793B) and Nobilis ${ }^{\circledR}$ IB Ma5 (IB-Ma5) (Intervet International B.V., BoxmeerHolland)).

\section{Chickens and the experimental design}

A total of 240-day-old birds (110 native breed Saudi chickens and 110 Ross breed) were obtained and distributed into 12 groups (20 birds each) (Table 1). Six groups out of the twelve were native Saudi chicken breeds. The other six groups of chickens were Ross foreign breeds. 
Table 1 Summary of the experimental design of the chicken experiment

\begin{tabular}{|c|c|c|c|c|c|c|c|}
\hline No & Group/Day & D-1 & D-7 & D-14 & D-21 & Challenge D-28* & \\
\hline 1 & $\mathrm{~B}-(-\mathrm{Ve})-\mathrm{CL}$ & No & NDV & IBDV & No & NDV/IBDV & No \\
\hline 2 & $\mathrm{R}(-\mathrm{Ve})-\mathrm{CL}$ & No & NDV & IBDV & No & NDV/IBDV & No \\
\hline 3 & $\mathrm{~B}-(+\mathrm{Ve})-\mathrm{CL}$ & No & NDV & IBDV & No & NDV/IBDV & $\mathrm{Y}$ \\
\hline 4 & $\mathrm{R}-(+\mathrm{Ve})-\mathrm{CL}$ & No & NDV & IBDV & No & NDV/IBDV & $\mathrm{Y}$ \\
\hline 5 & B-4-(+ Ve-IBV-vacc-1\&21d $)$ & IBV-4/91 & NDV & IBDV & IBV-MA5 & NDV/IBDV & $\mathrm{Y}$ \\
\hline 6 & R-4-(+ Ve-IBV-vacc-1\&21d) & IBV-4/91 & NDV & IBDV & IBV-MA5 & NDV/IBDV & $\mathrm{Y}$ \\
\hline 7 & B-1-(+ Ve-IBV-vacc-21d) & No & NDV & IBDV & MA5 & NDV/IBDV & $\mathrm{Y}$ \\
\hline 8 & R-1-(+ Ve-IBV-vacc-21d) & No & NDV & IBDV & MA5 & NDV/IBDV & $\mathrm{Y}$ \\
\hline 9 & B-2-(+ Ve-IBV-vacc-1\&21d $)$ & IBV-4/91 & NDV & IBDV & IBV-MA5 & NDV/IBDV & Y \\
\hline 10 & R-2-(+ Ve-IBV-vacc-1\&21d) & IBV-4/91 & NDV & IBDV & IBV-MA55 & NDV/IBDV & Y \\
\hline 11 & B-3-(+ Ve-IBV-vacc-1\&21d) & IBV-MA5 & NDV & IBDV & IBV-4/91 & NDV/IBDV & Y \\
\hline 12 & R-3-(+ Ve-IBV-vacc-1\&21d) & IBV-MA5 & NDV & IBDV & IBV-4/91 & NDV/IBDV & Y \\
\hline
\end{tabular}

No $=$ no vaccine, Yes $=$ challenge, $B=$ Baladi chicken, $\mathrm{R}=$ Ross chicken, $\mathrm{NDV}=$ Newcastle diseases virus vaccine, $\mathrm{IBDV}=$ Infectious bursal diseases vaccine

${ }^{*}$ Challenge at 28 days of age
Several hygienic and biosafety precautions were implemented to avoid any potential transmission of the IBV between various groups of birds during the tenure of these experiments., each group of birds were kept in a separate room with private feeders, heaters, and drinkers. The noninfected birds were kept in a separate wing of rooms apart from the challenged birds. These rooms and wings do not share a ventilation system. Each room had its own footbath filled with the proper disinfectants all the time. The noninfected birds usually approached first, then the challenged birds. The handling staff wore all the personal protective equipment, including disposable shoes and head covers as well as coats. These chickens received feed and water ad libitum throughout the tenure of the experiment. Two groups (one Ross and one native breed) received no vaccines and were not challenge with the IBV. Two groups (one Ross and one native breed) were challenged at 21 days of age but not received any IBV vaccines (Table 1). Two groups of chickens received IBV vaccines (4/91 and MA5) in a prime-boost regime at 14 and 21 days. While another two groups received the IBV vaccines (MA5 and 4/91) in a prime-boost regime at 14 and 21 days (Table 1).

\section{Serum samples collection and processing}

The serum samples of chickens were collected from the wing veins per each bird. The collected blood samples were processed by centrifugation at 5000 RPM. The supernatant clear sera were aspirated into another clean tube and stored at $4{ }^{\circ} \mathrm{C}$ for further testing. All serum samples were heat-inactivated at $56{ }^{\circ} \mathrm{C}$ for $30 \mathrm{~min}$ to remove the non-specific inhibitors.

\section{Challenging of the chickens with the IBV field isolate from eastern Saudi Arabia}

The inoculum was prepared from the laboratory confirmedpassage three of the ECE. Birds were challenged with $100 \mu \mathrm{l}$ of the virus suspension intranasal. The administration of the inoculum was conducted as previously described [13]. Birds were monitored three times daily for the development of any apparent clinical signs until the end of the experiment (birds at 48 days of age).

\section{Enzyme-linked immunosorbent assay (ELISA)}

We used the commercial ELISA kits (The IDEXX-IBV$\mathrm{Ab}$ test, IDEXX, USA) for testing the collected sera. We conducted the ELISA technique as per the manufacturer's instructions as wells previously described [12]. We used the average optical density values (OD) to calculate the S/P ratio then IBVantibodeis in sera of tested chickens under study using the standard equation provided by the manufactures. The data analysis was conducted as per the kit's instructions.

\section{Results}

\section{Description of the IBV infected chickens}

We examined one broiler chicken farm in eastern Saudi Arabia complaining of acute respiratory manifestations. The examined birds were showing classical respiratory signs, including sneezing, rales, rapid breathing, ruffled feather, diarrhea, and nasal discharges. Necropsy examination of some affected birds revealed the presence of 
caseous cheese like plugs in the tracheal bifurcations in addition to congestion and hemorrhage in the entire respiratory tract with consolidation in the lungs.

\section{Detection of some IBV circulating strains in eastern Saudi Arabia}

We tested some pooled tissue specimens from some birds that showed typical respiratory manifestations using the partial IBV-N gene primers. We were able to identify several positive birds by the RT-PCR, as showing in Fig. 1. Confirmation of the identity of the positive samples was revealed by the sequencing of the amplified PCR amplicons.

\section{Isolation of some filed strains of IBV from chickens in eastern Saudi Arabia}

We used some of the laboratory-confirmed IBV field isolates from eastern Saudi Arabia for the virus isolation via the embryonated chicken eggs (ECE). We performed three blind passages for virus isolation. We confirmed the presence of the IBV-RNAs in the tissues and fluids of the infected ECE after each passage. The pooled samples from the egg fluids and their tissues were used for the subsequent passages. The inoculated embryonated eggs showed some pathological changes in the form of dwarfing, hemorrhage, and poor feathering in the infected ECE (Fig. 2). Whereas the PBS negative control infected embryos showed normal size and appearance of the ECE (Fig. 2).

\section{Monitoring the clinical status of the IBV vaccinated and challenged birds}

We observed the experimental chicken three times daily for the development of any obvious clinical signs before and

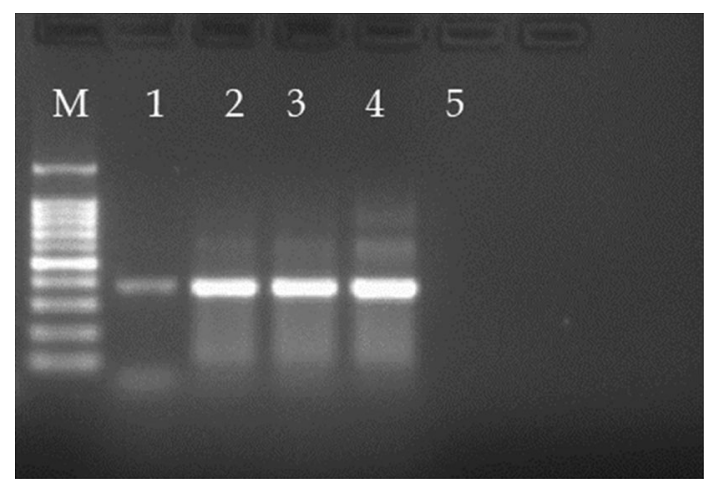

Fig. 1 Detection of the IBV in pooled tissues and organs of chickens in eastern Saudi Arabia by the RT-PCR using the partial IBV-N gene primers. The RT-PCR results of some pooled tissues (trachea, lung, kidney, spleen, and thymus gland). Lane $\mathrm{M}$ is the DNA ladder. Lanes 1-4 represent samples from five chickens. Lane 5 is empty well after the challenge. We also monitored the average body weight throughout the experiments (data not shown). We have not reported any apparent clinical manifestations, especially the respiratory signs in both the challenged birds as well as the negative control groups (Fig. 3). Both the challenged and the negative control groups of birds were of average body weight of chicken around the date of termination of the chicken experiment at 42 days of age.

\section{Monitoring the immune status of the IBV vaccinated and challenged birds}

The ELISA results are showing both two non-vaccinated, and none-challenged chicken groups (native chickens and Ross breed chicken) remained negative throughout the experiment. The IBV-antibody titers remained below the positive threshold values for the test (Fig. 4). The two none-IBV vaccinated and challenged birds seroconverted for the IBV, starting 35 days of age (Fig. 4). The primeboost IBV vaccinated and challenged a group of chickens started to seroconvert as early as one-week post-challenge (35 days of age). The native Saudi breeds had higher antibody titers than the Ross breeds at the end of the experiment (42 days of age) Fig. 4.

\section{Discussion}

The IBV is still posing a great risk to the poultry industry worldwide. This is due to the high rate of morbidity, mortality, poor feed conversion rate, as well as poor egg quality in layer chickens [7]. Several genotypes of various pathogenicity and virulence were reported recently in Saudi Arabia [9, 11, 12]. Although the IBV vaccines are administered to various categories of birds, either broiler or layers, several outbreaks are still reported worldwide $[14,15]$. The reason behind this may be due to the contains mutation and recombination among the circulating strains [16]. It is highly recommended that IBV vaccines should be prepared from at least homologous strains or from the recently isolated filed strains. The main goals for the current study were to identify the circulating strains of the IBV in some chicken populations in eastern Saudi Arabia than to isolate these strains via the ECE. To avoid any possible spread and carry over of the IBV from one group to another during the duration of this study, we adopted several hygienic and biosecurity measures. First, the negative control and the non-IBV challenged grup of birds were placed in a separate rooms and wings from the challenged birds. Seond, the handling personell adhered to all standared hyagenic measures inclusing wearing the personal protective equipments such as shoes and head coveres as well as disposable coats which they exchange before 
Fig. 2 Isolation of some circulating strains of IBV from chickens in eastern Saudi Arabia using embryonated chicken eggs, a First embryonated chicken egg passage, left: an embryonated chicken embryo inoculated with the IBV, right an embryonated chicken embryo inoculated with PBS. b Second embryonated chicken egg passage, left: an embryonated chicken embryo inoculated with the IBV, right an embryonated chicken embryo inoculated with PBS. c Third embryonated chicken egg passage, nine days old embryonated chicken embryos that inoculated with PBS showing the normal average size and feathering appearance. d nine days old embryonated chicken embryos inoculated with IBV showing dwarfing of the inoculated embryo
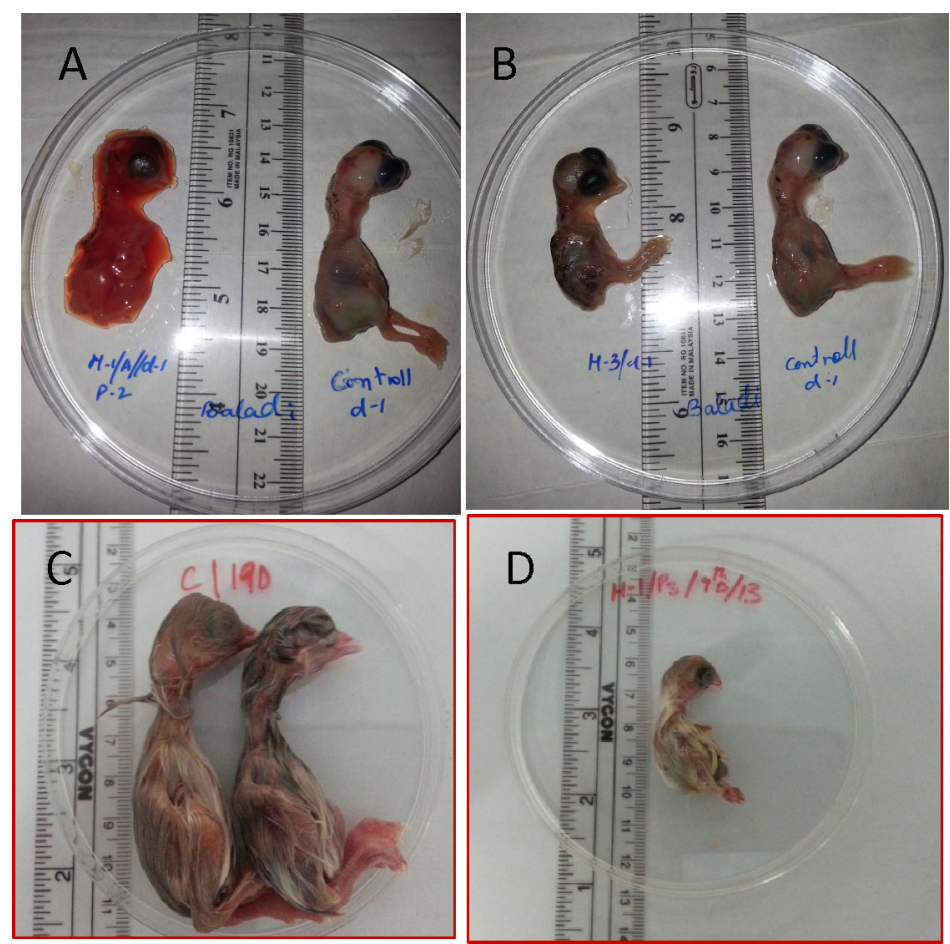

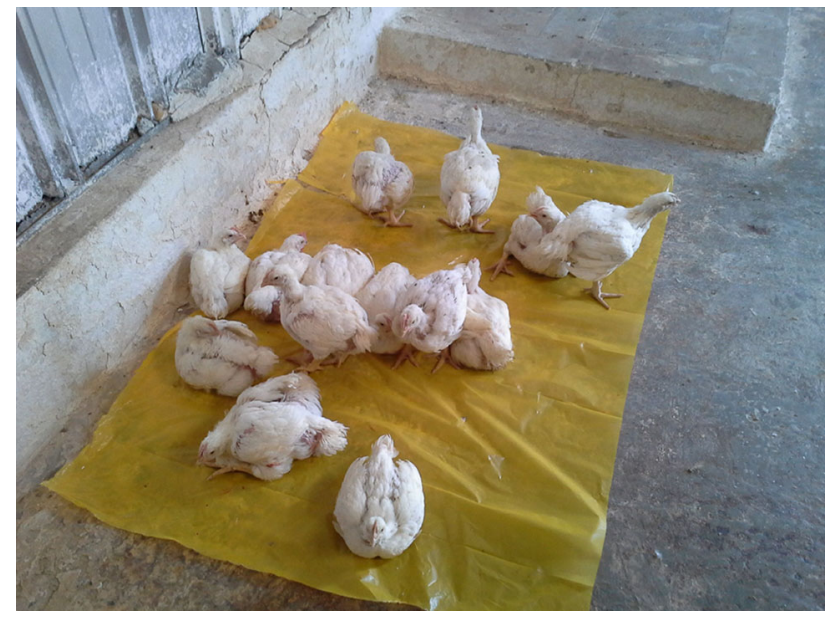

Fig. 3 Clinical appearance of the IBV challenged birds with the field strains. A group of challenged birds with the IBV field isolates from an outbreak in a farm in eastern Saudi Arabia at 45 days of age. Birds were apparently healthy, with no obvious signs of any respiratory distress

moving from one group to another. Third, each room had a separate own footbath. We tried to reproduce the IBV infection in some day-old native chicken Saudi breeds as well as some commercial chicken breeds such as the Ross chickens. We were able to isolate several isolates of the IBV using the ECE through three subsequent passages (Fig. 2). The inoculated ECE showed typical cytopathic effect produced by the IBV infection on the embryos in the form of congestion, hemorrhage, dwarfing and abnormal feather appearance of the embryos. These findings are consistent with the pathology induced by the virulent IBV infection in SPF chicken [17]. We harvested the egg fluids and the tissues and used them as inoculum for challenging purposes. All the challenged birds of both breeds did not show any IBV signs until the end of the experiment at 42 days of age (Fig. 3). The absence of any signs of diseases in the challenged birds may be attributed to the that more passages of the IBV field isolates in the ECE were required. We evaluated the immune status of the twelve groups of birds at $(1,7,14,21,28,35$, and 42$)$ days of age (Table 1). Both groups of birds which did not receive any vaccine or challenge remained seronegative for the IBV throughout the duration of the experiment (Fig. 4). Interestingly, the group of birds, which did not receive IBV vaccines but challenged showed seroconversion at 42 days of age. The native Saudi chicken breeds had higher antibody titres than the similar treated group of Ross chickens (Fig. 4). The other vaccinated birds through prime-boost regime and challenged showed the trend of slight antibody reduction after challenge with one week then the titre of the IBV antibody raised into double and three times in case of the Ross and native Saudi breeds respectively (Fig. 4). The prime-boost regime of vaccination against IBV with two different types of vaccines [18]. This is consistent with our data since the native breed chickens vaccinated with the IBV-4/91 then boosted with the IBV-MA5 showed higher antibody titres than the similar treated group of Ross chickens (Fig. 4). The lack of serconversion in the non- 


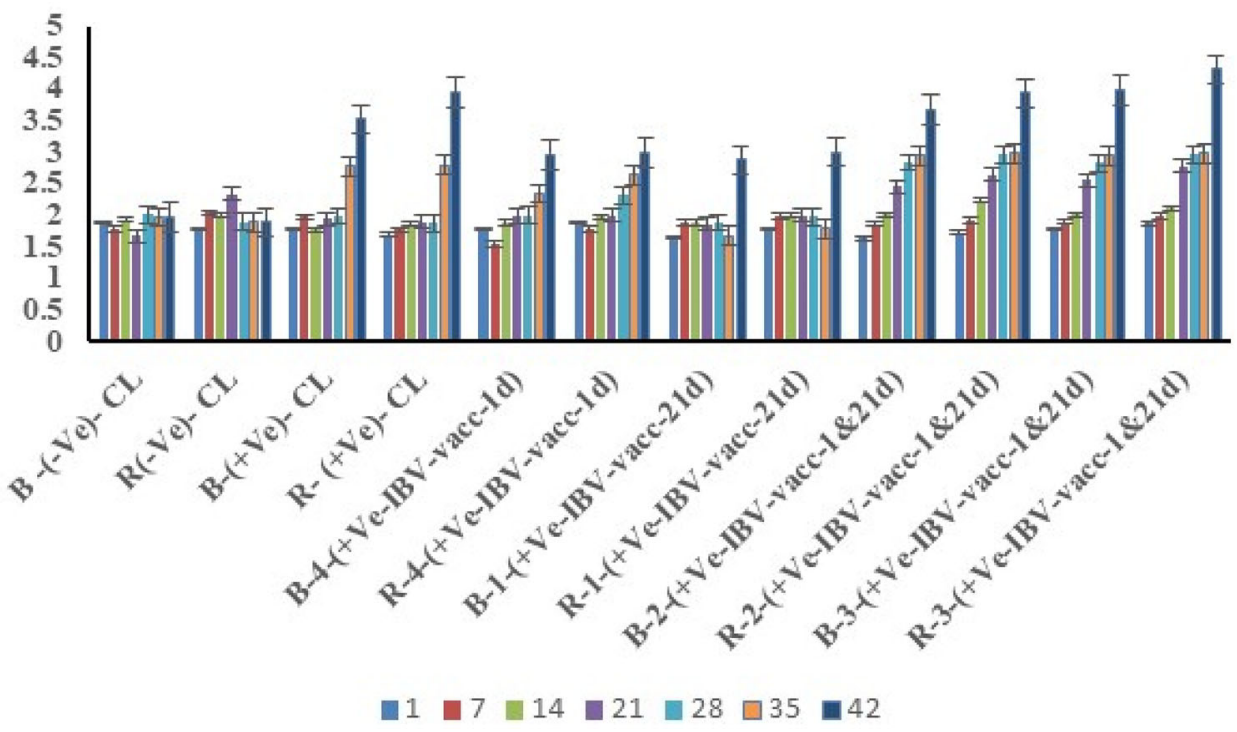

Fig. 4 Histogram of the ELISA results for the chicken experiment. Histogram showing the ELISA results of the chicken experiments. The antibody titers were obtained from ELISA for various groups of chickens at weekly intervals until the end of the chicken experiment. Some groups receive no vaccines and no challenge, while some other groups were challenged without receiving any vaccines. Other groups

challnges-nonvaccinated group of birds indicates the lack of any carry over of theIBV between various groups of birds. Further studies are required for a better understanding of the circulating IBV strains in chickens at the eastern Saudi as well as to understand the dynamics of the of the humoral immunity for the IBV in the native chicken as well as other commercial breeds.

Acknowledgments This work was supported by the King Faisal University Deanship of research Grant No. (142017). We also than Mr. Ahmed Alkhars with his laboratory technical helps during the virus isolation and pathogenicity index.

\section{Compliance with ethical standards}

Conflict of interest All authors declare no conflict of interest.

\section{References}

1. Cavanagh D. Coronaviruses in poultry and other birds. Avian Pathol. 2005;34(6):439-48.

2. Fellahi S, et al. Prevalence and molecular characterization of avian infectious bronchitis virus in poultry flocks in Morocco from 2010 to 2014 and first detection of Italy 02 in Africa. Avian Pathol. 2015;44(4):287-95.

3. Fraga AP, et al. Phylodynamic analysis and molecular diversity of the avian infectious bronchitis virus of chickens in Brazil. Infect Genet Evol. 2018;61:77-83.

4. Rohaim MA, et al. Emergence and genetic analysis of variant pathogenic 4/91 (serotype 793/B) infectious bronchitis virus in Egypt during 2019. Virus Genes. 2019;55(5):720-5. were received prime and boost vaccines for the IBV. The histogram showing birds received a double dose of vaccines (prime and boost) seroconverted to the IBV at 42 days of age. The challenged birds which did not receive any vaccines also seroconverted to the IBV around 35 days of age

5. Zhang X, et al. Molecular characterization of variant infectious bronchitis virus in China, 2019: implications for control programmes. Transbound Emerg Dis. 2020;67(3):1349-55.

6. Masters PS. The molecular biology of coronaviruses. Adv Virus Res. 2006;66:193-292.

7. Jackwood MW. Review of infectious bronchitis virus around the world. Avian Dis. 2012;56(4):634-41.

8. Guy JS. Isolation and propagation of coronaviruses in embryonated eggs. Methods Mol Biol. 2015;1282:63-71.

9. Alsultan MA, Alhammadi MA, Hemida MG. Infectious bronchitis virus from chickens in Al-Hasa, Saudi Arabia 2015-2016. Vet World. 2019;12(3):424-33.

10. Hemida MG, Alnaeem A. Some One Health based control strategies for the Middle East respiratory syndrome coronavirus. One Health. 2019;8:100102.

11. Al-Mubarak AIA, Al-Kubati AAG. Cocirculation of Four infectious Bronchitis Virus Lineages in Broiler Chickens in the Eastern Region of Saudi Arabia from 2012 to 2014. Vet Med Int. 2020;2020:6037893.

12. Hemida MG, et al. Molecular characterization and phylogenetic analyses of virulent infectious bronchitis viruses isolated from chickens in Eastern Saudi Arabia. Virusdisease. 2017;28(2):189-99.

13. Sultan HA, et al. Protective Efficacy of Different Live Attenuated Infectious Bronchitis Virus Vaccination Regimes Against Challenge With IBV Variant-2 Circulating in the Middle East. Front Vet Sci. 2019;6:341.

14. Jordan B. Vaccination against infectious bronchitis virus: a continuous challenge. Vet Microbiol. 2017;206:137-43.

15. Roussan DA, Khawaldeh GY, Shaheen IA. Infectious bronchitis virus in Jordanian chickens: seroprevalence and detection. Can Vet J. 2009;50(1):77-80.

16. Nix WA, et al. Emergence of subtype strains of the Arkansas serotype of infectious bronchitis virus in Delmarva broiler chickens. Avian Dis. 2000;44(3):568-81. 
17. Li YT, et al. Emerging lethal infectious bronchitis coronavirus variants with multiorgan tropism. Transbound Emerg Dis. 2020;67(2):884-93.

18. Yan F, et al. Protection of chickens against infectious bronchitis virus with a multivalent DNA vaccine and boosting with an inactivated vaccine. J Vet Sci. 2013;14(1):53-60.
Publisher's Note Springer Nature remains neutral with regard to jurisdictional claims in published maps and institutional affiliations. 